\title{
Formación del Profesorado y Demostración Matemática. Estudio Exploratorio e Implicaciones
}

\author{
Teacher Training and Mathematical Proof. \\ Exploratory Study and Implications
}

\author{
Alberto Arnal-Bailera* \\ Antonio M. Oller-Marcén **
}

\begin{abstract}
Resumen
En el presente trabajo se aborda el diseño y análisis de una actividad de formación de profesorado de secundaria orientada a analizar el conocimiento sobre la demostración (en el marco del TPACK) de futuros docentes de matemáticas de secundaria en formación. En particular, observamos que los alumnos tienen un buen conocimiento del contenido (CK), así como un buen conocimiento tecnológico (TK), pero aparecen dificultades al introducir la componente pedagógica y, en particular, las interrelaciones entre todas ellas. Estos resultados permiten, finalmente, señalar algunas líneas de actuación en torno a las que organizar el trabajo sistemático de la demostración en el aula con futuros docentes a nivel de máster.
\end{abstract}

Palabras clave: Formación del profesorado. TPACK. Demostración matemática. GeoGebra.

\begin{abstract}
In this paper, we address the design and analysis of a teacher training activity about proof focussed on the analysis of the knowledge (through TPACK) of prospective mathematics secondary school teachers. In particular, we observe that the students have a good knowledge of the content (CK), as well as a good knowledge of the technology (TK). Some difficulties are found when the pedagogical component is introduced and when the interrelations among all of them are considered. These results allow us to point out some action lines in order to organise the systematic work on proof with prospective mathematics teachers in their master's degree.
\end{abstract}

Keywords: Teacher training. TPACK. Mathematical proof. GeoGebra.

\section{Introducción y objetivos}

Diversos autores (SCHOENFELD, 1994; HANNA, 1995) ponen de manifiesto la necesidad y el interés de trabajar la demostración en el aula, no sólo por su importancia desde el punto de vista del quehacer matemático, sino por su contribución a la comprensión de los

\footnotetext{
* Doctor en Didáctica de las Matemáticas por la Universidad Autónoma de Barcelona (UAB). Profesor en la Universidad de Zaragoza (UZ), Zaragoza, España. Dirección postal: C/Pedro Cerbuna 12; 50009, Zaragoza, Zaragoza, España. E-mail: albarnal@unizar.es.

** Doctor en Didáctica de las Matemáticas por la Universidad de Valladolid (UVA). Profesor en el Centro Universitario de la Defensa de Zaragoza (CUDZ), Zaragoza, España. Dirección postal: Ctra. de Huesca, s/n; 50090, Zaragoza, Zaragoza, España. E-mail: oller@unizar.es.
} 
conceptos matemáticos involucrados. Esta necesidad ha sido recogida, de forma desigual, en los distintos planes de estudios españoles desde 1934 (IBAÑES; ORTEGA, 2002). Estos mismos autores señalan que, pese a algunas deficiencias, ya la Ley Orgánica de Ordenación General del Sistema Educativo - LOGSE supuso una mejora muy importante respecto a planes de estudios anteriores. Algunas de las deficiencias indicadas se solventaron en la Ley Orgánica de Educación - LOE, finalmente, la Ley Orgánica para la Mejora de la Calidad Educativa - LOMCE establece el aprendizaje de la demostración como obligatorio y transversal a todos los contenidos (CONEJO, 2015).

Sin embargo, los contenidos relativos a la demostración se presentan en forma de lista, sin un orden lógico que ayude al profesor a planificar su enseñanza. Sólo aparecen dos criterios de evaluación, relativo uno a la demostración de propiedades o teoremas, y el otro a la comunicación de con rigor de ideas matemáticas presentes en esas demostraciones. Por último, los estándares de aprendizaje asociados valoran el uso de diversos métodos, la reflexión sobre el proceso seguido, el lenguaje matemático, la argumentación y el empleo de herramientas tecnológicas. En todo caso, el currículo no incluye indicaciones metodológicas concretas que expliquen cómo llevar al aula los contenidos de este bloque o lo diferencien de los demás; que resultarían de ayuda en su implementación práctica.

A consecuencia de lo anterior, surge el interés de llevar a cabo actividades de formación de profesorado de secundaria relacionadas con la demostración, que involucren tanto aspectos relativos a la práctica de la demostración, como a su enseñanza y aprendizaje. Así pues, abordamos el diseño de una actividad de formación de profesorado de secundaria cuyos objetivos son:

- Analizar el conocimiento de los estudiantes del Máster de profesorado de Secundaria acerca de la práctica de la demostración en Matemáticas.

- Obtener implicaciones para la mejora de la docencia del propio Máster.

\section{Marco teórico}

Shulman (1986) presentaba la idea de conocimiento pedagógico del contenido (PCK) que ha resultado de utilidad a la hora de diseñar y analizar actividades de formación del profesorado. Hoy en día, el trabajo en el aula pasa por la necesaria inclusión de herramientas tecnológicas (incluso obligada en ocasiones por el propio currículo oficial). Así pues, los componentes fundamentales de una adecuada enseñanza no serían ya solo el contenido y la 
pedagogía, sino que debe incluirse la tecnología al mismo nivel que las anteriores (KOEHLER; MISHRA, 2009). Esta perspectiva (Technological Pedagogical Content Knowledge - TPACK) es consistente y extiende la anterior ocupándose además de las complejas relaciones entre los factores señalados.

En la Figura 1 se presenta un esquema de los dominios y subdominios considerados por el marco TPACK, que describimos a continuación.

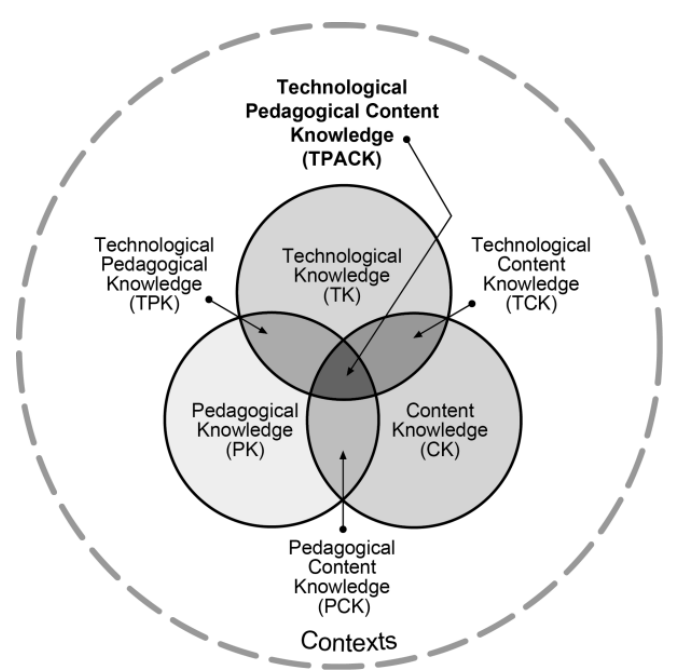

Figura 1 - Esquema del contenido pedagógico tecnológico (TPACK) Fuente: http://tpack.org

Donde las siglas significan:

- CK: Conocimiento del contenido matemático del profesor, incluyendo conceptos, teorías, ideas, marcos de organización etc.

- PK: Conocimiento del profesor de los procesos, prácticas y métodos de enseñanza y aprendizaje de las matemáticas.

- PCK: Conocimiento del profesor sobre las posibilidades de transformación o adaptación del contenido matemático para la enseñanza.

- TK: Conocimiento del profesor que le permite realizar diversas tareas utilizando las tecnologías de la información y de encontrar diferentes formas de realizar una tarea dada.

- TCK: Conocimiento del profesor sobre cómo la tecnología y el contenido se influencian y limitan mutuamente.

- TPK: Conocimiento del profesor sobre los cambios que el uso de la tecnología genera en la enseñanza y el aprendizaje.

Cada situación de enseñanza de las matemáticas hace emerger una combinación nueva de los tres factores (contenido, pedagogía y tecnología). Para evitar la simplificación de las 
respuestas educativas o el fracaso en su aplicación, los profesores deben conocer además las interrelaciones entre estos tres dominios y cómo un cambio en uno de ellos afecta a los otros dos.

Mouza et al. (2014) señalan la necesidad de trabajar los distintos dominios del modelo a la hora de abordar la formación del profesorado. Otras investigaciones que siguen el planteamiento propuesto en el modelo TPACK concluyen también "la conveniencia de potenciar la capacitación y perfeccionamiento en TIC en la formación inicial de los maestros" (CÓZAR; ZAGALAZ; SÁEZ, 2015, p. 163).

Por otro lado, debemos tener en cuenta que la tecnología es únicamente un instrumento curricular, cuyo uso tendrá sentido en función de las relaciones que se establezcan entre el mismo y los restantes componentes del currículum (CABERO, 2010). A partir de la descripción realizada anteriormente, se observa que el marco TPACK pone su énfasis en las interrelaciones existentes entre los tres factores primarios (contenido, pedagogía y tecnología) y no tanto en cada uno de ellos individualmente. Por ello, se trata de un marco especialmente adecuado, en nuestra opinión, para el trabajo efectivo con profesorado en formación.

Hemos comentado, en la introducción, que ya desde la LOGSE la demostración aparece de manera clara en el currículum español. Ante esta presencia de la demostración en el aula, trabajos como (DOS SANTOS; ORTEGA, 2013) ponen de manifiesto la gran diversidad de posturas existentes dentro del profesorado en ejercicio respecto a diversos aspectos de la demostración. Por su parte, investigaciones como la de Crespo y Ponteville (2005) indican que la demostración en el aula suele ignorarse, o bien se presenta con una función de verificación y, en menor medida, de explicación.

Las funciones de sistematización, descubrimiento o comunicación (DE VILLIERS, 1993) apenan aparecen. Incluso encontramos trabajos (VICARIO; CARRILLO, 2005) que ponen de manifiesto posibles limitaciones de algunos profesores a la hora de comprender ciertas demostraciones, como las realizadas por reducción al absurdo. Por otro lado, Montoro (2007) muestra que la demostración se concibe por parte del profesorado en formación como un conocimiento principalmente procedimental, que se adquiere mediante la repetición y la práctica. En este mismo sentido, el trabajo de Samper, Ospina y Plazas (2010) muestra cómo, entre el profesorado universitario de Matemáticas, existe la idea de que la enseñanza de la demostración se debe hacer presentado ejemplos de demostraciones y que su aprendizaje se logra principalmente haciendo demostraciones y examinando ejemplos. 
La demostración en Geometría, con ayuda de un programa de geometría dinámica, ha sido estudiada por diversos investigadores. Por ejemplo, Marrades y Gutiérrez (2000) distinguen entre justificaciones empíricas y deductivas. Las primeras, que pueden clasificarse en: justificación naïf (basada en el estudio particular de uno o varios casos, elegidos sin criterio alguno, en los que la propiedad a demostrar es cierta), justificación mediante experimento (basada en el estudio particular de un caso elegido cuidadosamente para ser considerado lo menos particular posible) y justificación mediante ejemplo genérico (basada en el estudio de un ejemplo específico representativo, pero incluyendo razones abstractas para justificar la validez de la conjetura mediante operaciones o transformaciones del ejemplo). Las segundas se caracterizan por la descontextualización de los argumentos y se clasifican según si para organizar la demostración se usa un ejemplo (experimento reflexionado) o no (formal).

\section{Método y muestra}

La experimentación se llevó a cabo con 11 estudiantes (7 graduados en Matemáticas, 1 en Física y 3 en Ingeniería) del Máster en Profesorado de Educación Secundaria Obligatoria (especialidad de Matemáticas), durante el curso 2014-2015, y en el marco de la asignatura Diseño, organización y desarrollo de actividades para el aprendizaje de Matemáticas. En concreto, la actividad se desarrolló en una sesión de dos horas de duración en el aula de informática. Durante la sesión se alternó el trabajo individual o en parejas (codificadas como C1 a C7) de los alumnos con las puestas en común coordinadas por los investigadores.

Para el diseño del cuestionario (ver Anexo) se optó por un contexto de Geometría plana elemental. En particular, las tareas propuestas están relacionadas con las Proposiciones 18, 19 y 20 del Libro I de los Elementos y se presentaban, a modo de información, la definición de triángulo isósceles y las Proposiciones 5 y 16 de ese mismo Libro I. Los contenidos matemáticos implicados involucran relaciones entre la amplitud de los ángulos y la longitud de los lados en un triángulo.

Pensamos que los contenidos elegidos suponen un contexto adecuado para abordar una actividad relacionada con la demostración, tanto por su relativa sencillez desde el punto de vista matemático, como por la clara secuenciación lógica de los mismos y los pocos conocimientos previos necesarios (proporcionados a modo de información). De las múltiples ediciones de los Elementos, optamos por la muy curiosa de Oliver Byrne (1847) en la que el 
sistema de representación elegido para nombrar los objetos geométricos se basa en el uso de diversos colores (TUFTE, 1998) y tipos de trazos. El uso de esta heterodoxa edición de un más heterodoxo autor (BARNETT, 2010) resulta interesante, entre otros aspectos, para introducir un cierto relativismo en el aula, así como para enfatizar la dimensión humana de la actividad matemática; aspectos que Jahnke et al. (2000) señalan, entre otros, como motivos para introducir el uso de fuentes históricas.

Las cinco tareas planteadas a los alumnos se corresponden con los distintos dominios y subdominios considerados en el marco TPACK. En concreto:

- En la tarea 1 se solicita a los estudiantes que completen dos demostraciones con las justificaciones que consideren adecuadas, utilizando las informaciones básicas proporcionadas. Esta tarea se corresponde con el conocimiento del contenido (CK).

- En la tarea 2 se solicita una reflexión sobre las dos demostraciones presentadas en la tarea $1 \mathrm{y}$, en particular, se pretende que los estudiantes comparen dos modos distintos de abordar una demostración. Esta tarea se enmarca en el conocimiento pedagógico del contenido (PCK).

- La tarea 3 implica la reescritura de una demostración para que se ajuste al procedimiento de reducción al absurdo. Nuevamente se enmarca en el conocimiento pedagógico del contenido (PCK).

- En la tarea 4 los estudiantes deben adaptar la demostración de la proposición trabajada en la tarea 3 para su hipotética presentación en el aula, utilizando GeoGebra. Esta tarea se corresponde con el conocimiento pedagógico de la tecnología (TCK).

- En la tarea 5 los estudiantes debían planificar una actividad relacionada con la demostración. Esta tarea se enmarca en el conocimiento pedagógico y tecnológico del contenido (TPACK).

El estudio realizado es exploratorio, con una finalidad esencialmente descriptiva (ELLIOT; TIMULAK, 2005). La recogida de información se ha abordado mediante la utilización de tres procedimientos. En primer lugar, la observación por parte de los investigadores. En segundo lugar, las producciones de los alumnos participantes, plasmadas en sus respuestas al cuestionario (tareas 1 a 5) y en los ficheros de GeoGebra generados en la tarea 4. Finalmente, en tercer lugar, las fases de puesta en común (tareas 1, 2 y 3 ) fueron grabadas en video y transcritas para su posterior análisis. Esta triangulación de los datos (DENZIN, 1978) contribuye a mejorar la calidad de la investigación (ONWUEGBUZIE; LEECH, 2007). 


\section{Resultados}

\subsection{Tarea 1 - Apartado A}

En la tarea 1a (Figura 2) se pide la inclusión de las justificaciones que consideren necesarias en los distintos pasos de la demostración de la Proposición 18.

TAREA 1. Completa las siguientes demostraciones con las justificaciones que consideres oportunas. Puedes utilizar los hechos matemáticos anteriores.

PROPOSICIÓN 18: En cualquier triángulo
es mayor que otro
lado

Figura 2 - Enunciado y pasos de la demostración presentada en la tarea 1a Fuente: elaboración propia

En el Cuadro 1 se indica, para cada uno de los pasos en los que se descompone la demostración de esta proposición, si la pareja correspondiente ha proporcionado o no algún tipo de justificación o comentario.

\begin{tabular}{|l|c|c|c|c|c|c|c|}
\hline & C1 & C2 & C3 & C4 & C5 & C6 & C7 \\
\hline Paso 1 & NO & NO & SI & NO & NO & SI & SI \\
\hline Paso 2 & NO & SI & SI & SI & SI & SI & SI \\
\hline Paso 3 & SI & SI & SI & NO & SI & SI & SI \\
\hline Paso 4 & NO & SI & NO & NO & SI & NO & NO \\
\hline Paso 5 & NO & NO & NO & NO & SI & NO & NO \\
\hline
\end{tabular}

Cuadro 1- Justificaciones dadas por las parejas de alumnos para la tarea 1a. Fuente: elaboración propia

En el paso 1 cuatro parejas no incluyen ningún tipo de comentario, dando por hecho que es posible realizar la construcción indicada y no señalando cómo se hace. La pareja C6 indica el proceso necesario para llevar a cabo la construcción; las otras dos parejas (C3 y C7) resaltan que se obtiene un triángulo isósceles. 
En el paso 2 todas las parejas salvo $\mathrm{C} 1$ incluyen algún tipo de comentario. El resto de las parejas hacen uso de la Proposición 5, pero solo dos de ellas (C4 y C5) lo indican explícitamente.

En el paso 3, tres de las parejas (C3, C6 y C7) justifican haciendo referencia explícita a la proposición 16 que se les había aportado. Dos parejas (C1 y C2) obvian esta información previa, incluyen simbolismo algebraico y razonan planteando ecuaciones y/o inecuaciones. La pareja C4 no incluye ningún comentario mientras que la pareja restante (C5) introduce un argumento dinámico basado en la variación simultánea de dos de los ángulos de un triángulo cuando el otro se mantiene fijo (Figura 3), podemos leer la argumentación de los alumnos: $S i$ prolongamos el lado rojo el ángulo opuesto es más grande (dibujo) El ángulo que forman rojo y azul no varía. Entonces para que sigan sumando $180^{\circ}$ el ángulo amarillo tiene que ser más pequeño que el azul.

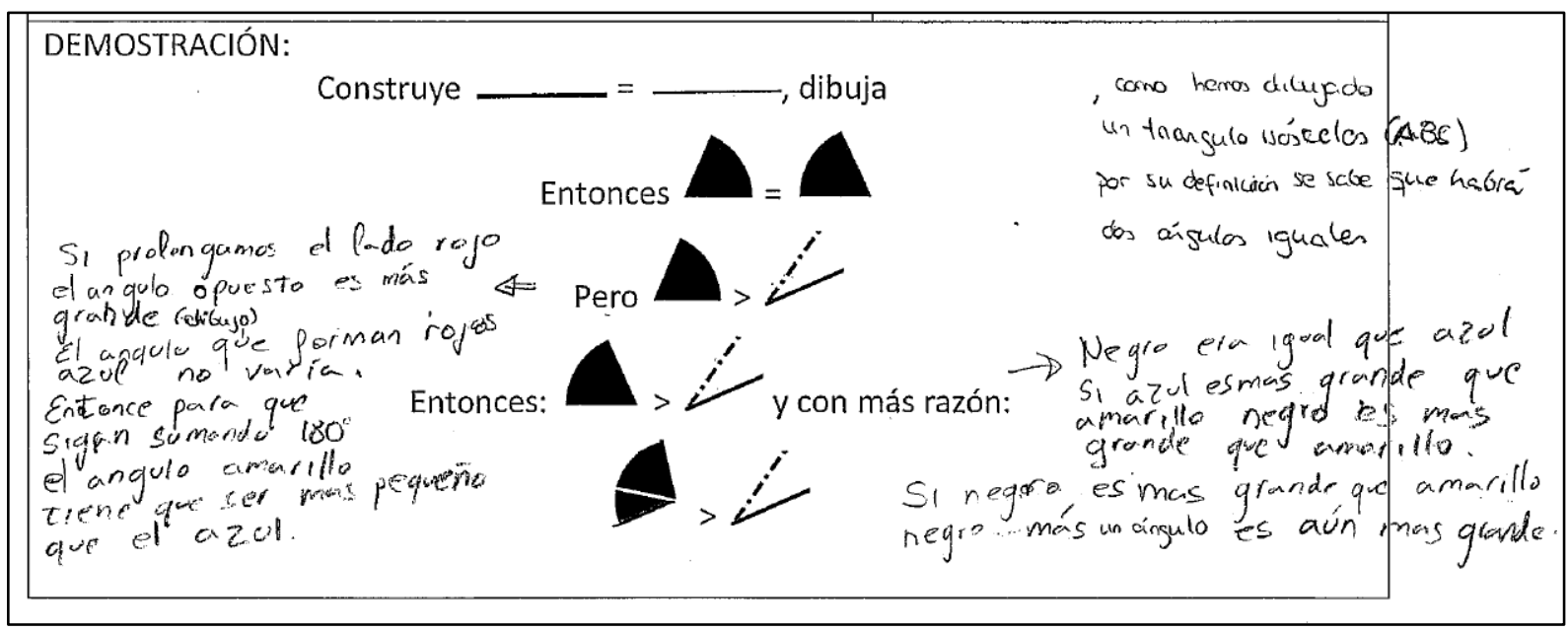

Figura 3 - Resolución de la tarea 1a por la pareja C5

Fuente: elaboración propia

En el paso 4 solo una de las parejas (C5) aplica la propiedad transitiva entre igualdades y desigualdades. Una pareja (C2) hace una justificación incompleta y el resto no dan ninguna explicación al respecto.

En el paso 5 cinco de las parejas no realizan ningún comentario. Del resto, una de ellas (C5), explicita una justificación y otra (C1) se limita a cambiar a un sistema de representación simbólico.

\subsubsection{Tarea 1 - Apartado B}


En la tarea 1b (Figura 4) se pide la inclusión de las justificaciones que consideren necesarias en los distintos pasos de la demostración de la Proposición 19.

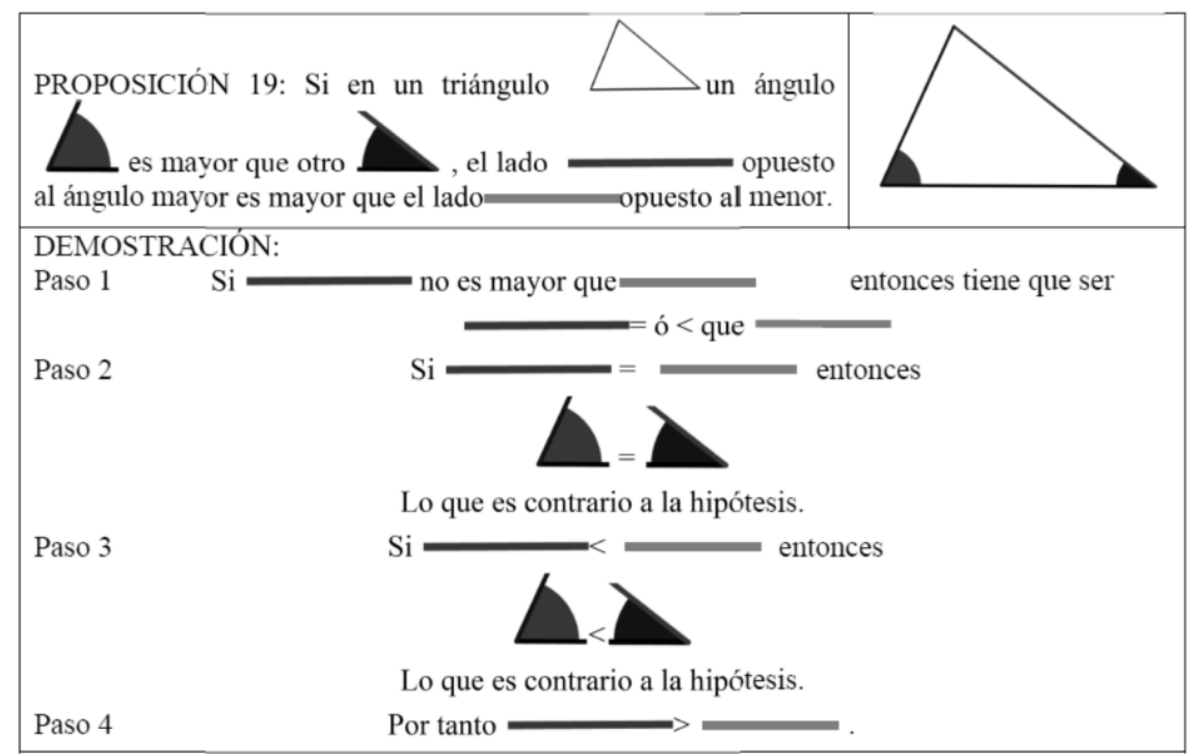

Figura 4 - Pasos de la demostración presentada en la tarea 1b Fuente: elaboración propia

En el Cuadro 2 se indica, para cada uno de los pasos en los que se descompone la demostración de esta proposición, si la pareja correspondiente ha proporcionado o no algún tipo de justificación o comentario.

\begin{tabular}{|l|l|l|l|l|l|l|l|}
\hline & C1 & C2 & C3 & C4 & C5 & C6 & C7 \\
\hline Paso 1 & NO & NO & NO & NO & NO & NO & NO \\
\hline Paso 2 & SI & SI & SI & SI & NO & SI & SI \\
\hline Paso 3 & NO & SI & SI & SI & SI & SI & SI \\
\hline Paso 4 & NO & NO & NO & NO & NO & NO & NO \\
\hline
\end{tabular}

Cuadro 2 - Justificaciones dadas por las parejas de alumnos para la tarea $1 \mathrm{~b}$ Fuente: elaboración propia

En el paso 1 ninguna pareja incluye comentario alguno, dando por hecho no solo la veracidad de la afirmación sino su carácter auto-explicativo.

En el paso 2, solamente una de las parejas (C5) no da ninguna justificación, aunque la justificación de otra pareja (C1) no tiene sentido. El resto de las parejas hacen uso de la Proposición 5, pero solo tres de ellas (C2, C3 y C4) lo indican explícitamente, mientras que C5, C6 y C7 hacen un uso implícito de esta Proposición (Figura 5).

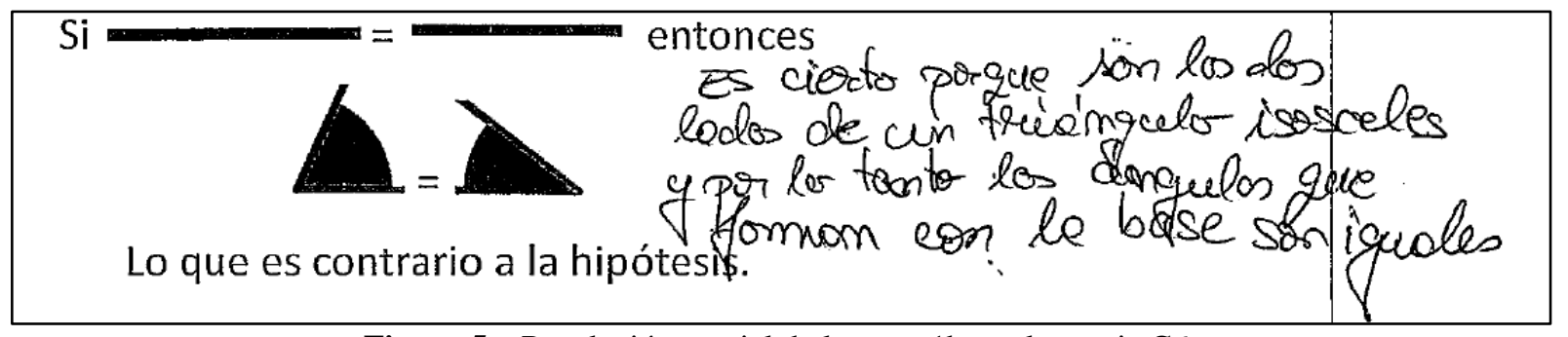

Figura 5 - Resolución parcial de la tarea 1b por la pareja C6 


\section{Fuente: elaboración propia}

En el paso 3, la pareja C1 no da ninguna justificación. El resto las dan basándose explícitamente en la Proposición 18.

El paso 4 no es justificado por ninguna pareja.

En la puesta en común de la tarea 1, se hizo énfasis en aquellos pasos que no habían recibido justificación por parte de las parejas participantes. Como se observa en los cuadros anteriores, se trata fundamentalmente de aquellos pasos que involucran aspectos que pueden considerarse evidentes (paso 5 del apartado a) o principios lógicos comúnmente utilizados (paso 1 del apartado b). Algunas parejas señalan que estos son aspectos que para un matemático son evidentes, pero que requerirían explicación para una persona de letras.

\subsection{Tarea 2}

En esta tarea se pide comentar las diferencias entre las formas de razonar empleadas en las demostraciones anteriores (ver Figura 6).

TAREA 2 ¿Aprecias alguna diferencia entre las formas de razonar que se emplean en las dos demostraciones anteriores? Coméntalas.

\section{Figura 6 - Enunciado de la tarea 2 Fuente: elaboración propia}

En la Cuadro 3 se indica si la pareja correspondiente ha dado alguna denominación a cada demostración, proporcionando o no algún tipo de justificación al nombre, y si ha realizado comentarios sobre la dificultad o accesibilidad de ambas demostraciones para los alumnos de bachillerato.

\begin{tabular}{|c|c|c|c|c|c|c|c|c|}
\hline & $\mathrm{C} 1$ & $\mathrm{C} 2$ & C3 & $\mathrm{C} 4$ & $\mathrm{C} 5$ & C6 & $\mathrm{C} 7$ \\
\hline \multicolumn{2}{|c|}{ Realiza la tarea } & $\mathrm{NO}$ & SI & SI & $\mathrm{SI}$ & SI & SI & SI \\
\hline \multirow[b]{2}{*}{ P.18 } & Denominación & N/A & Constructiva & Constructiva & Deductiva & Deductiva & Deductiva & Constructiva \\
\hline & $\begin{array}{l}\text { Razona la } \\
\text { denominación }\end{array}$ & N/A & SI & SI & SI & $\mathrm{NO}$ & SI & SI \\
\hline \multirow{2}{*}{ P.19 } & Denominación & N/A & $\begin{array}{l}\text { Reducción } \\
\text { al absurdo }\end{array}$ & $\begin{array}{l}\text { Reducción } \\
\text { al absurdo }\end{array}$ & $\begin{array}{l}\text { Reducción } \\
\text { al absurdo }\end{array}$ & $\begin{array}{l}\text { Reducción } \\
\text { al absurdo }\end{array}$ & $\begin{array}{l}\text { Reducción } \\
\text { al absurdo }\end{array}$ & $\begin{array}{l}\text { Reducción } \\
\text { al absurdo }\end{array}$ \\
\hline & $\begin{array}{l}\text { Razona la } \\
\text { denominación }\end{array}$ & N/A & $\mathrm{NO}$ & SI & SI & NO & SI & SI \\
\hline \multicolumn{2}{|c|}{$\begin{array}{l}\text { Comentarios sobre } \\
\text { dificultad o } \\
\text { accesibilidad }\end{array}$} & N/A & NO & NO & $\mathrm{NO}$ & NO & $\mathrm{NO}$ & NO \\
\hline
\end{tabular}

Cuadro 3 - Análisis de las respuestas escritas dadas por los alumnos en la tarea 2 Fuente: elaboración propia

Todas las parejas salvo una $(\mathrm{C} 1)$ realizan la tarea. 
Respecto de la demostración de la Proposición 18, esta es denominada como deductiva por tres de las parejas que realizan la tarea $(\mathrm{C} 4, \mathrm{C} 5$ y C6). Las otras tres la califican como constructiva (Cuadro 3). Estos calificativos son justificados por todas las parejas salvo una (C5).

Respecto de la demostración de la Proposición 19, esta es denominada como reducción al absurdo por todas las parejas que realizan la tarea. Dos parejas no justifican esta denominación (C2 y C5), de entre las parejas que lo justifican, dos no lo hacen de un modo completamente correcto ( $\mathrm{C} 3$ y $\mathrm{C} 7$ ) ya que indican que se debe llegar a contradicción con la hipótesis del enunciado (Figura 7).

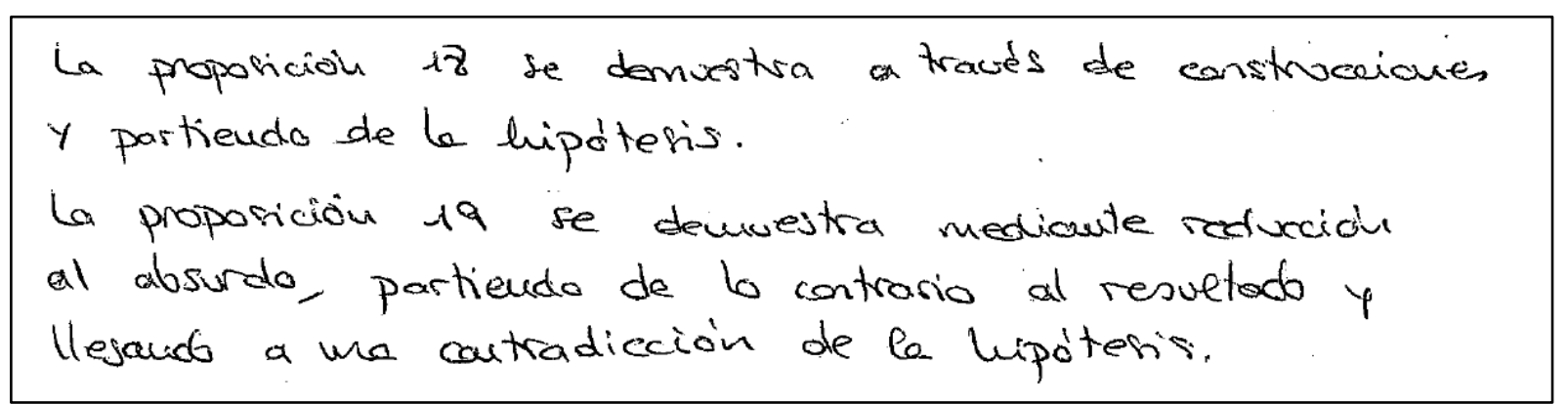

Figura 7 - Resolución de la tarea 2 por la pareja C7

Fuente: elaboración propia

Ninguna pareja incluye entre las diferencias ninguna referencia a la dificultad matemática o a la accesibilidad de estos tipos de razonamiento para sus futuros alumnos o para ellos mismos.

La puesta en común de esta tarea se centró en cuestiones relacionadas con la dificultad o accesibilidad de cada uno de los dos tipos de demostraciones. Existe un consenso en que ambas demostraciones son igualmente convincentes y satisfactorias, pero que para los alumnos es más difícil de comprender la demostración por reducción al absurdo. Algunas parejas señalan que la reducción al absurdo se utilizaría en el aula en última instancia pues su carácter es más profesional. Otra pareja señala que la demostración directa debe presentarse antes porque implica construir y llega a calificar esta demostración directa de demostración normal. En cualquier caso, todos los intervinientes parecen coincidir en que las dificultades de la reducción al absurdo radican en la necesidad de un cierto manejo de la lógica y la comprensión de ideas como la negación, los contra-ejemplos etc.

\subsection{Tarea 3}


En esta tarea se les proporciona la demostración de la Proposición 20 y se pide reescribirla mediante reducción al absurdo (Figura 8).

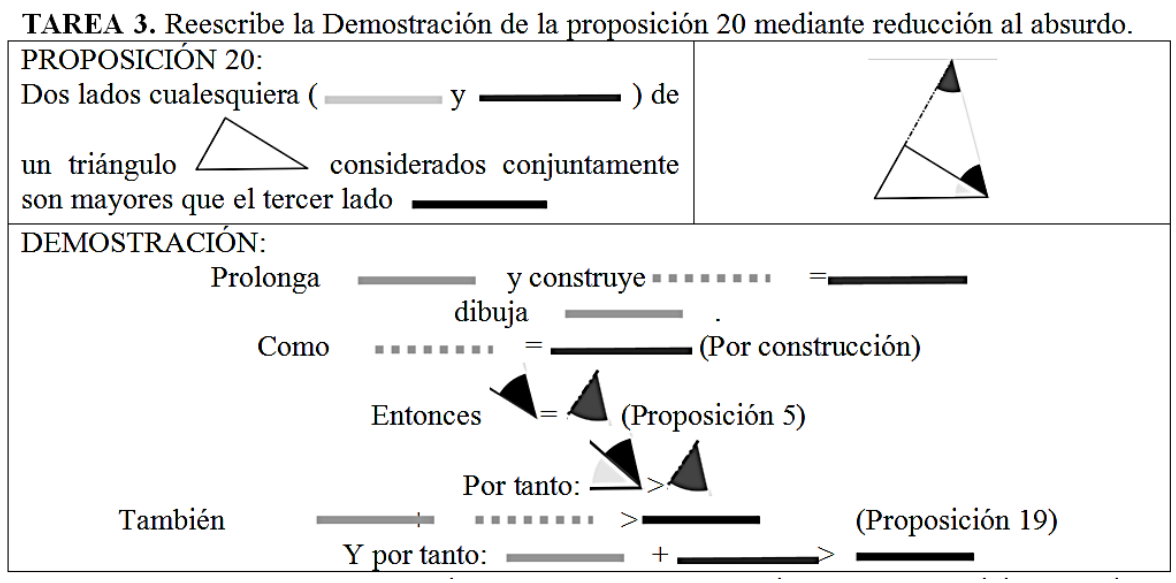

Figura 8 - Enunciado de la tarea 3

Fuente: elaboración propia

En el Cuadro 4 se indica si la pareja correspondiente ha realizado correctamente la tarea, si ha traducido la demostración original y si ha introducido o no notación simbólica.

\begin{tabular}{|l|l|l|l|l|l|l|l|}
\hline & C1 & C2 & C3 & C4 & C5 & C6 & C7 \\
\hline Realiza correctamente la tarea & NO & SI & NO & SI & SI & SI & SI \\
\hline Traduce la demostración original & N/A & NO & N/A & SI & SI & SI & NO \\
\hline Introduce notación simbólica & N/A & SI & N/A & SI & SI & SI & NO \\
\hline
\end{tabular}

Cuadro 4- Análisis de las respuestas dadas por los alumnos en la tarea 3 Fuente: elaboración propia

Todas las parejas salvo dos $(\mathrm{C} 1$ y $\mathrm{C} 3)$ realizan la tarea completamente. De entre aquellas que realizan la tarea, dos (C2 y $\mathrm{C} 7)$ no realizan una traducción de la demostración original, sino que la abordan de forma completamente diferente. Las otras tres parejas realizan la demostración partiendo de la negación de la tesis y alcanzando dos contradicciones que, unidas a la utilización implícita del principio del tercio excluso dan lugar a la veracidad de la tesis (ver Figura 9).

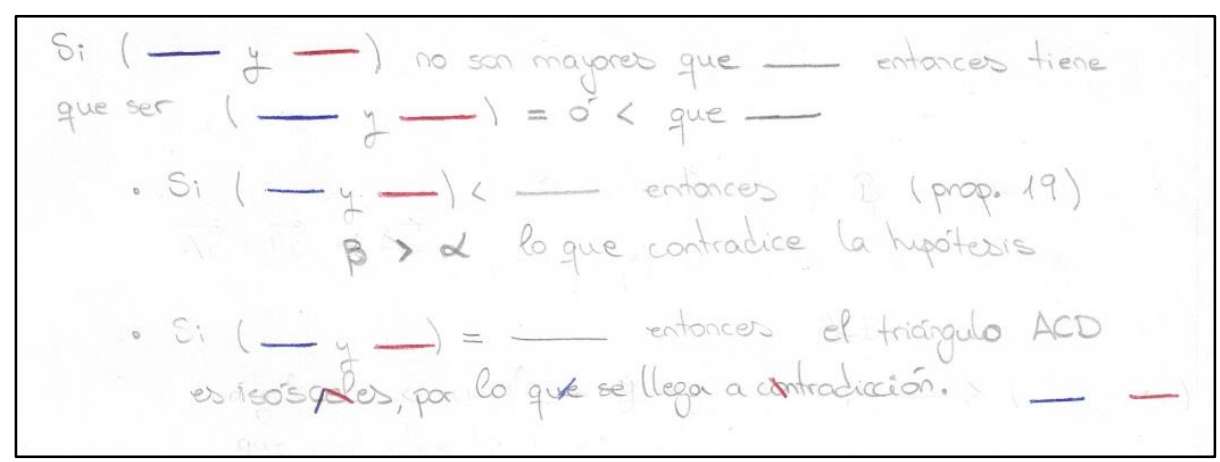

Figura 9 - Resolución de la Tarea 3 por la pareja de alumnos C4 Fuente: elaboración propia 
Es interesante observar que todas las parejas, salvo una (C7), introducen notación simbólica en mayor o menor medida en sus producciones (ver Figura 10).

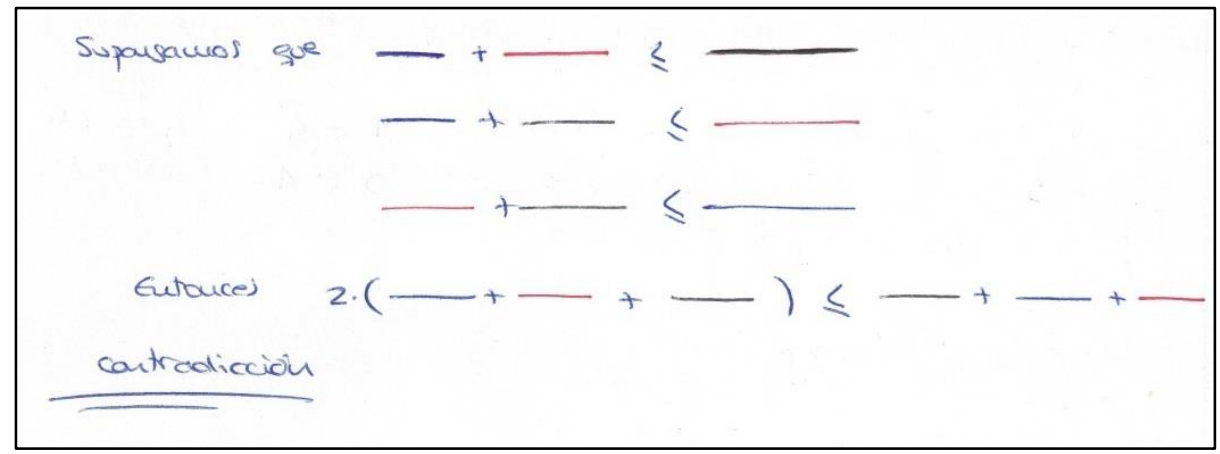

Figura 10 - Resolución de la Tarea 3 por la pareja de alumnos C7

Fuente: elaboración propia

En la puesta en común no se dan demasiadas intervenciones. Algunas parejas tienen dificultades para explicar verbalmente el proceso seguido y salen a la pizarra para poder apoyar su discurso por escrito. El recurso al lenguaje simbólico se justifica por parte de algunas parejas en que se sienten más cómodas en el mundo del álgebra que de la geometría.

\subsection{Tarea 4}

En esta tarea (Figura 11) se pide que construyan una animación con GeoGebra con el propósito de utilizarla como apoyo de una supuesta explicación verbal que tuvieran que dar en clase de la demostración de la Proposición 20. Además, deben aportar un guión de la intervención. En el Cuadro 5 se indica si la pareja correspondiente ha realizado la construcción con GeoGebra y ha elaborado guiones para su uso.

TAREA 4: Supón que debes explicar en clase la demostración de la proposición anterior. Construye una animación con GeoGebra y utilízala para apoyar tu explicación verbal. Escribe un guión de tu intervención en el que se muestre cómo combinas el uso del software con tus explicaciones.

\section{Figura 11 - Enunciado de la tarea 4}

Fuente: elaboración propia

Todas las parejas, salvo una (C2), realizan una construcción con GeoGebra. En uno de los casos (C1) la construcción aportada no se corresponde con la demostración de la Proposición 20 que se aportaba a los alumnos.

\begin{tabular}{|l|l|l|l|l|l|l|l|}
\hline & C1 & C2 & C3 & C4 & C5 & C6 & C7 \\
\hline Construcción GeoGebra & SI & NO & SI & SI & SI & SI & SI \\
\hline Correspondencia con la demostración aportada & NO & N/A & SI & SI & SI & SI & SI \\
\hline Guión tecnológico (cuestiones sobre la construcción) & NO & NO & SI & NO & SI & NO & SI \\
\hline Guión pedagógico (cuestiones sobre el uso de la construcción) & SI & SI & NO & SI & SI & SI & NO \\
\hline Uso por el profesor del GGB & SI & SI & SI & SI & SI & SI & SI \\
\hline
\end{tabular}




\begin{tabular}{|l|l|l|l|l|l|l|l|l|}
\hline Uso por los alumnos el GGB & NO & NO & NO & NO & NO & SI & NO \\
\hline
\end{tabular}
Fuente: elaboración propia

Todas las parejas escriben un guión, si bien varias de ellas (C3 y C7) incluyen solamente referencias al proceso de construcción de GeoGebra, sin hacer referencias precisas a cómo usarlo en la práctica de aula (guión tecnológico). Otras parejas (C1, C2, C4 y C6) incluyen referencias solamente al uso de la animación con alumnos (guión pedagógico) sin hacer referencia a detalles acerca de la propia construcción con GeoGebra. La pareja C5 es la única que incluye comentarios de ambas categorías (Figura 12).

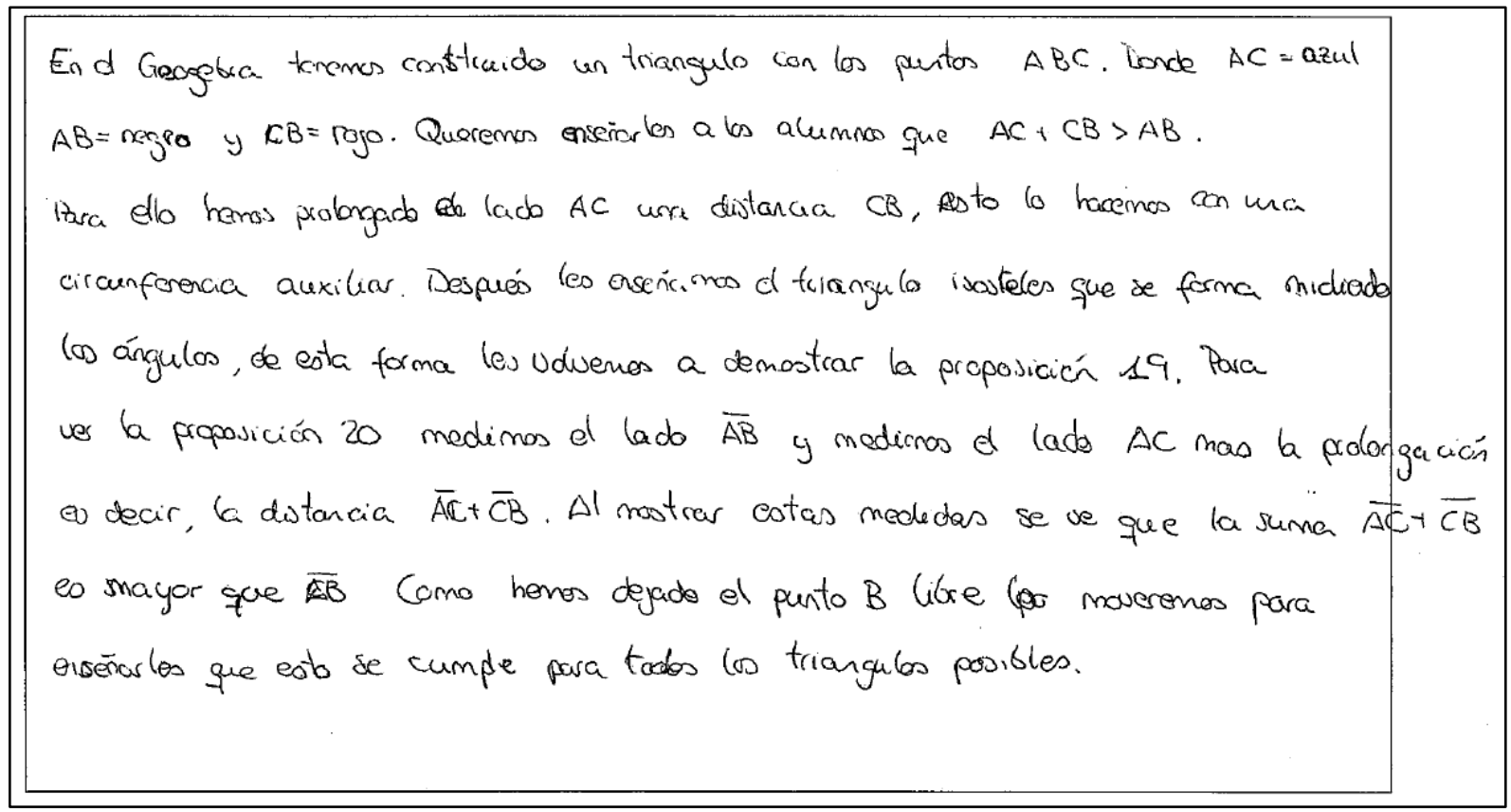

Figura 12 - Resolución de la Tarea 4 por la pareja de alumnos C5

Fuente: elaboración propia

Sólo una pareja (C6) plantea que sean los alumnos los que utilicen la animación para explorar el caso en que la suma de las longitudes de dos lados coincida con la longitud del tercero y vean que en ese caso no hay triángulo. El resto de las parejas limitan implícitamente el apoyo a la explicación al uso por parte del profesor ya que no se refieren a los alumnos en sus guiones.

El análisis de los ficheros GeoGebra generados por las parejas participantes muestra que todas las parejas, menos dos, utilizan las funcionalidades dinámicas del programa. Sin embargo, al analizar el guión pedagógico - cuando aparece - observamos que en la mayor parte de los casos es el profesor - ...vamos obteniendo diferentes triángulos - y no el alumno quien hace uso de dichas funcionalidades - los alumnos manipulan GeoGebra hasta 
obtener...-. En el primer caso GeoGebra se utiliza de un modo similar al que se utilizaría en una pizarra tradicional para apoyar el discurso.

\subsection{Tarea 5}

En esta tarea se pide que diseñen una actividad relacionada con la demostración e indiquen cómo la evaluarían (Figura 13).

TAREA 5. Centrándote en algún aspecto concreto del currículo de Bachillerato (Álgebra, Análisis...), diseña una actividad relacionada con la demostración e indica cómo la evaluarías

\section{Figura 13 - Enunciado de la tarea 5}

Fuente: elaboración propia

Solamente cuatro de las parejas realizan esta última tarea (C1, C3, C5 y C6).

Tres de las parejas (C1, C3 y C5) proponen, exclusivamente, la demostración de una propiedad. La otra pareja (C6) que realiza la tarea propone, aunque sea esquemáticamente, una actividad doble consistente en hacer una exploración con GeoGebra para encontrar el rectángulo de área máxima para un perímetro dado y después demostrarlo a través del estudio de los máximos de una función (Figura 14).

$$
\begin{aligned}
& \text { - ¿'Gual es el rectaingulo de cirea méxima cuyo prínutoo } \\
& \text { el } 2004 \text { ? } \\
& \text { (1) En geogebra. } \\
& \text { (2) Intentar la dewartración a través del atudio }
\end{aligned}
$$

Figura 14 - Resolución de la Tarea 4 por la pareja de alumnos C5 Fuente: elaboración propia

Respecto de la evaluación, dos de las parejas realizan una propuesta (C3 y C5) dando puntos por conocer los teoremas o propiedades implicados en la demostración (ambos) o expresarse correctamente (C5).

\begin{tabular}{|l|l|l|l|l|l|l|l|}
\hline & C1 & C2 & C3 & C4 & C5 & C6 & C7 \\
\hline Realiza la tarea & SI & NO & SI & NO & SI & SI & NO \\
\hline La actividad consiste solamente en demostrar una propiedad & SI & N/A & SI & N/A & SI & NO & N/A \\
\hline Propone evaluación & NO & N/A & SI & N/A & SI & NO & N/A \\
\hline
\end{tabular}

Cuadro 6 - Análisis de las respuestas escritas dadas por los alumnos en la tarea 5 Fuente: elaboración propia

\section{Discusión e implicaciones}


La tarea 1 exigía que los participantes pusieran en juego su conocimiento matemático, justificando los pasos de dos demostraciones. Hemos observado que no se justifican todos los pasos con la misma frecuencia. En particular, aquellos que involucran únicamente el uso de un razonamiento lógico elemental (en opinión de los participantes) no suelen ser justificados. Sin embargo, aquellos que suponen la aplicación de resultados matemáticos anteriores raramente quedan sin justificar. El carácter, elemental o no, de un razonamiento lógico debería ser percibido por parte de los futuros docentes como fuertemente dependiente de los receptores potenciales de la demostración. Este hecho iría en perjuicio de las funciones (DE VILLIERS, 1993) de explicación y comunicación de la demostración y debería ser tenido en cuenta a la hora de planificar las actividades con profesorado de secundaria en formación.

En la tarea 2, donde se solicitaba comparar las dos demostraciones anteriores, sí observamos una cierta preocupación por el alumno y por las dificultades que este puede encontrar según el tipo de demostración. Perciben la reducción al absurdo como más complicada para los alumnos, posiblemente como un reflejo de sus propias dificultades con este tipo de demostración (VICARIO; CARRILLO, 2005). Además, observamos que el conocimiento y el uso del lenguaje especializado, en este contexto, es cuando menos impreciso en el sentido de Dos Santos y Ortega (2013). Esto puede apuntar a que los profesores de secundaria en formación necesitarían ampliar sus conocimientos sobre las distintas funciones de la demostración para que perciban el posible interés de trabajar la reducción al absurdo con sus futuros alumnos, puesto que llegan a calificar la demostración directa como normal. No hay que olvidar que el nuevo currículum de Secundaria incluye, entre sus contenidos, diversos métodos de demostración (reducción al absurdo, inducción etc.)

Las dificultades mencionadas con la reducción al absurdo se vuelven a poner de manifiesto en la tarea 3 cuando los participantes deben reescribir una demostración utilizando dicha técnica. De hecho, más de la mitad de los participantes no realizaron de manera satisfactoria esta tarea, fundamentalmente aquellos con una menor formación matemática a nivel de Grado. Una de las manifestaciones más claras de esta dificultad es el recurso al lenguaje algebraico, cuando ni el contexto de la tarea ni el sistema de representación utilizado inducían a ello. De nuevo, esto puede ocasionar problemas en la comunicación con los alumnos y que las dificultades que los alumnos de secundaria muestran en los contenidos algebraicos se extiendan a otros bloques de contenido como la geometría (ARNALBAILERA, 2013). Este tipo de tareas de adaptación o reescritura pueden ser por tanto de interés para el profesorado de secundaria en formación. 
Aun reconociendo la importancia de la tecnología en la enseñanza y el aprendizaje de las matemáticas, los profesores de secundaria en formación muestran dificultades en su aplicación cuando deben conectarla con el contenido y la pedagogía (MOUZA et al., 2014). Este hecho se pone de manifiesto tanto en la tarea 4 (por el uso dado a GeoGebra) como en la 5 (por la escasa presencia del software).

Pese a que, como señala Cabero (2010, p. 48), una de las principales características de los entornos tecnológicos de formación es la interactividad de los mismos, se observa el predominio de los usos centrados en el profesor jugando GeoGebra, un papel simplemente de apoyo a la explicación y obviando los otros usos propuestos por Lasa y Wilhelmi (2013). Hay que indicar que, en el momento de la experimentación, los participantes ya habían recibido instrucción sobre el manejo GeoGebra si bien desde el punto de vista matemático. Esto nos indicaría la necesidad de hacer énfasis en los usos pedagógicos de GeoGebra y no sólo de los meramente matemáticos. De hecho, como ya señalan Roblizo y Cózar (2015, p. 36) esto es especialmente necesario si tenemos en cuenta que, en la actualidad, gran parte del trabajo de un docente "pasa por la creación, evaluación y/o adaptación de recursos digitales a su práctica".

Por último, como indican autores como Montoro (2007) o Samper, Ospina y Plazas (2010) observamos una fuerte concepción procedimental de la demostración en la tarea 5 en la que los participantes debían diseñar una actividad relacionada con la demostración centrada en un contenido de Bachillerato. Todas las propuestas consistían, esencialmente, en demostrar un resultado concreto. No hay mención a distintos métodos, funciones o técnicas de demostración. La elaboración de actividades para el aula es una de las labores más importantes de un docente y la falta de variedad en las propuestas es indicadora de una deficiencia en la formación en este aspecto. Esta debilidad implica que los futuros docentes recurren a "conocimientos, creencias, actitudes, hábitos y conductas previos [...] a imitación de ciertas prácticas de enseñanza de sus antiguos profesores” tal y como indica Palarea (2011, p. 231).

El trabajo a nivel de Máster debería tener uno de sus focos principales en dotar a los futuros docentes de las herramientas necesarias para diseñar e implementar actividades ricas relacionadas, en este caso, con la demostración. El diseño de este tipo de tareas contribuirá, sin duda, a su profesionalización puesto que implica enfrentar a los futuros docentes a problemas y cuestiones que se plantearán en el futuro desempeño de su profesión (FLORES, 1998). 


\section{Conclusión}

En este trabajo hemos abordado el diseño y análisis de una actividad de formación de profesorado de secundaria centrada en la demostración matemática. Se perseguían dos objetivos, fundamentalmente. El primero, analizar el conocimiento (según los diversos dominios del marco TPACK) de los futuros docentes y, el segundo, obtener algunas implicaciones útiles para el trabajo con estos futuros docentes a nivel de máster.

En cuanto al primer objetivo, observamos que los futuros docentes tienen un buen conocimiento del contenido (CK), así como un buen conocimiento tecnológico (TK). Sin embargo, aparecen más problemas cuando entra en juego la componente pedagógica y, en particular, las interrelaciones entre ellas. De hecho, los resultados obtenidos en la tarea 5 que potencialmente implicaba una puesta en juego de todos estos tipos de conocimientos, ilustran claramente estos problemas.

Por último, respecto al segundo objetivo, los resultados obtenidos sugieren diversas líneas de actuación en torno a las que organizar el trabajo sistemático de la demostración en el aula con futuros docentes a nivel de máster. Por ejemplo:

- Deben presentarse las diversas funciones de la demostración, así como poner de manifiesto la necesidad de abordar y utilizar todas ellas en el aula.

- Los trabajos de adaptación y/o reescritura de demostraciones cambiando el método de demostración, variando el nivel de los posibles destinatarios etc. pueden ser actividades interesantes y formativas para los futuros docentes.

- Debe fomentarse la utilización de GeoGebra orientado a los alumnos y no sólo a los profesores.

- Los futuros docentes han de ser conscientes de que trabajar la demostración con alumnos de secundaria o bachillerato no debe limitarse a la mera demostración de resultados concretos.

\section{Agradecimientos}

Este trabajo ha sido desarrollado por el grupo de investigación S119-Investigación en Educación Matemática, financiado por el Gobierno de Aragón y el Fondo Social Europeo. También fue parcialmente financiado por el Ministerio de Economía y Competitividad de España (Proyecto EDU2015-65378-P). 


\section{Referencias}

ARNAL-BAILERA, A. Mediación Tecnológica en la Enseñanza y el Aprendizaje de Geometría con Grupos de Riesgo. 2013. 318 p. Tesis (doctorado en Didáctica de la Matemática) - Universidad Autónoma de Barcelona, Barcelona, 2013. Disponible en: 〈http://ddd.uab.cat/record/117881/〉.

Acceso en: 7 de abril de 2016.

BARNETT, J. H. Mathematics is a plural noun: the singular case of Oliver Byrne. ANNUAL MEETINGLREUNION ANNUELLE, 23., 2010, Ontario. Proceedings of the Canadian Society for the History and Philosophy of Mathematics, Ontario: CSHPM/SCHPM, 2010. p. 26-46.

BYRNE, O. The First Six Books of the Elements of Euclid in Which Coloured Diagrams and Symbols are Used Instead of Letters for the Greater Ease of Learners. 1. ed. London: William Pickering, 1847. 396 p.

CABERO, J. Los retos dela integración de las TICs en los procesos educativos. Límites y posibilidades. Perspectiva Educacional, Formación de Profesores, Valparaíso, v. 49, n. 1, p. 32-61, 2010.

CONEJO, L. Análisis Histórico de las Demostraciones en Libros de Texto Sobre los Teoremas de Límites y Continuidad. De la Ley General de Educación a la Ley Orgánica de Educación. 2015. 380 p. Tesis (doctorado en Didáctica de la Matemática) - Universidad de Valladolid, Valladolid, 2015. Disponible en: 〈https://www.educacion.es/teseo/mostrarRef.do?ref=1196001>. Acceso en: 7 de abril de 2016.

CÓZAR, R.; ZAGALAZ, J.; SÁEZ, J. Creando contenidos curriculares digitales de Ciencias Sociales para Educación Primaria. Una experiencia TPACK para futuros docentes. Educatio Siglo XXI, Murcia, v. 33, n.3, p. 147-168, 2015.

CRESPO, C. R.; PONTEVILLE, C. C. Las funciones de la demostración en el aula de matemática. Acta Latinoamericana de Matemática Educativa, México D.F., v.18, p. 307-312, 2005.

DE VILLIERS, M. El papel y la función de la demostración en matemáticas. Épsilon, Cádiz, n. 26, p. 15-30, 1993.

DENZIN, N. K. (1978). The Research Act: a theoretical introduction to sociological methods. 1. ed. New York: Praeger. 306 p.

DOS SANTOS, C.; ORTEGA, T. Perfiles del profesorado sobre la enseñanza y uso de la demostración. Avances de Investigación en Educación Matemática, Madrid, n. 4, p. 27-45, 2013.

ELLIOT, R.; TIMULAK, L. Descriptive and interpretive approaches to qualitative research. In: MILES, J.; GILBERT, P. (Ed.). A Handbook of Research Methods for Clinical and Health Psychology. New York: Ed. Oxford University Press, 2005. p. 147-159.

FLORES, P. Formación inicial de profesores de matemáticas como profesionales reflexivos. UNO. Revista de Didáctica de las Matemáticas, Barcelona, v. 17, p. 37-48, 1998.

HANNA, G. Challenges to the Importance of Proof. For the Learning of Mathematics, Ottawa, v. 15, n. 3, p. 42-49, 1995. 
IBAÑES, M.; ORTEGA, T. La demostración en el currículo: una perspectiva histórica. SUMA, Badalona, n. 39, p. 53-61, 2002.

JAHNKE, H. N. et al. The use of original sources in the mathematics classroom. In: FAUVEL, J.; Van MAANEN, J. (Ed.). History in mathematics education: the ICMI study. Dordrecht: Ed. Kluwer, 2000. p. 291-328.

KOEHLER, M. J.; MISHRA, P. What is technological pedagogical content knowledge?

Contemporary Issues in Technology and Teacher Education, Charlottesville, v. 9, n. 1, p. 60-70, 2009.

LASA, A.; WILHELMI, M. R. Use of GeoGebra in explorative, explanatory and demonstrative moments. Revista do Instituto GeoGebra Internacional de São Paulo, São Paulo, v. 2, n. 1, p. 5264, 2013.

MARRADES, R.; GUTIÉRREZ, Á. Proofs produced by secondary school students learning geometry in a dynamic computer environment. Educational Studies in Mathematics, Ámsterdam, v. 44, n. 1-2, p. $87-125,2000$.

MOUZA, C. et al. Investigating the impact of an integrated approach to the development of preservice teachers' technological pedagogical content knowledge (TPACK). Computers \& Education,

Ámsterdam, n. 71, p. 206-221, 2014.

MONTORO, V. Concepciones de estudiantes de profesorado acerca del aprendizaje de la demostración. Revista Electrónica de Investigación en Educación en Ciencias, Buenos Aires, v. 2, n. 1, p. 101-121, 2007.

ONWUEGBUZIE, A. J.; LEECH, N. L. Validity and qualitative research: an oxymoron? Quality \& Quantity, Berlín, v. 41, n. 2, p. 233-249, 2007.

PALAREA, M. Informe del seminario: la formación inicial del profesorado de matemáticas ante la implantación de los nuevos grados en infantil, primaria y máster de secundaria. Educatio Siglo XXI, Murcia, v. 29, n. 2, p. 225-234, 2011.

ROBLIZO, M. C.; CÓZAR, R. Usos y competencias en TIC de los futuros maestros de educación infantil y primaria: hacia una alfabetización tecnológica real para docentes. Pixel-Bit. Revista de Medios y Educación, Sevilla, n. 47, p. 23-39, 2015.

SAMPER, C.; OSPINA, Y. T.; PLAZAS, T. J. Aproximación a las visiones de demostración de algunos profesores universitarios de matemáticas. Tecné, Episteme y Didaxis, Bogotá, n. 27, p. 2537, 2010.

SCHOENFELD, A. What do we know about curricula? Journal of Mathematical Behavior, Ámsterdam, v. 13, n. 1, p. 55-80, 1994.

SHULMAN, L. S. Those who understand: knowledge growth in teaching. Educational Researcher, Boston, v. 15, n. 2, p. 4-14, 1986.

TUFTE, E. R. Envisioning Information. 1. ed. Cheshire, Connecticut: Graphic Press, 1998. 190 p.

VICARIO, V.; CARRILLO, J. Concepciones del profesor de secundaria sobre la demostración matemática. El caso de la irracionalidad de $\sqrt{ } 2$ y las funciones de la demostración. In: MAZ, A.; GÓMEZ, B.; TORRALBO, M. (Ed.). SIMPOSIO DE LA SOCIEDAD ESPAÑOLA DE 
INVESTIGACIÓN EN EDUCACIÓN MATEMÁTICA, 9., 2005. Actas del 9 Simposio de la SEIEM, Córdoba: SEIEM, 2005. p. 145-152.

Submetido em Abril de 2016. Aprovado em Outubro de 2016. 


\section{Anexo}

\section{TAREAS PARA LOS ALUMNOS}

Algunos hechos matemáticos acerca de los triángulos (tomados de los Elementos de Euclides, versión de Oliver Byrne 1857):

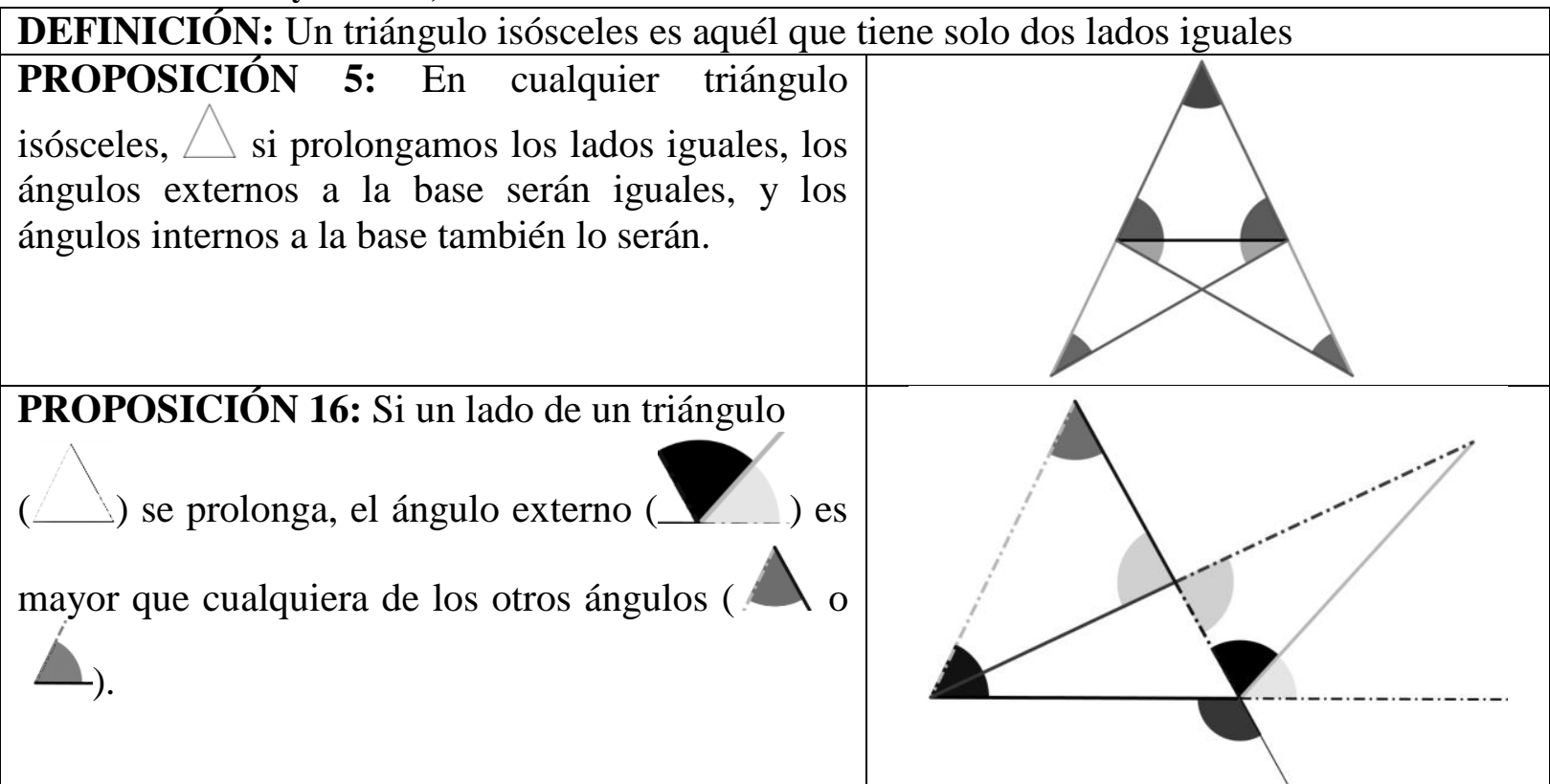

TAREA 1. Completa las siguientes demostraciones con las justificaciones que consideres oportunas. Puedes utilizar los hechos matemáticos anteriores.

PROPOSICIÓN 18: En cualquier triángulo
lado es mayor que otro
mayor es mayor que el ángulo opuesto al menor. Es
Paso 2
Paso 3
Paso 4
Paso 5




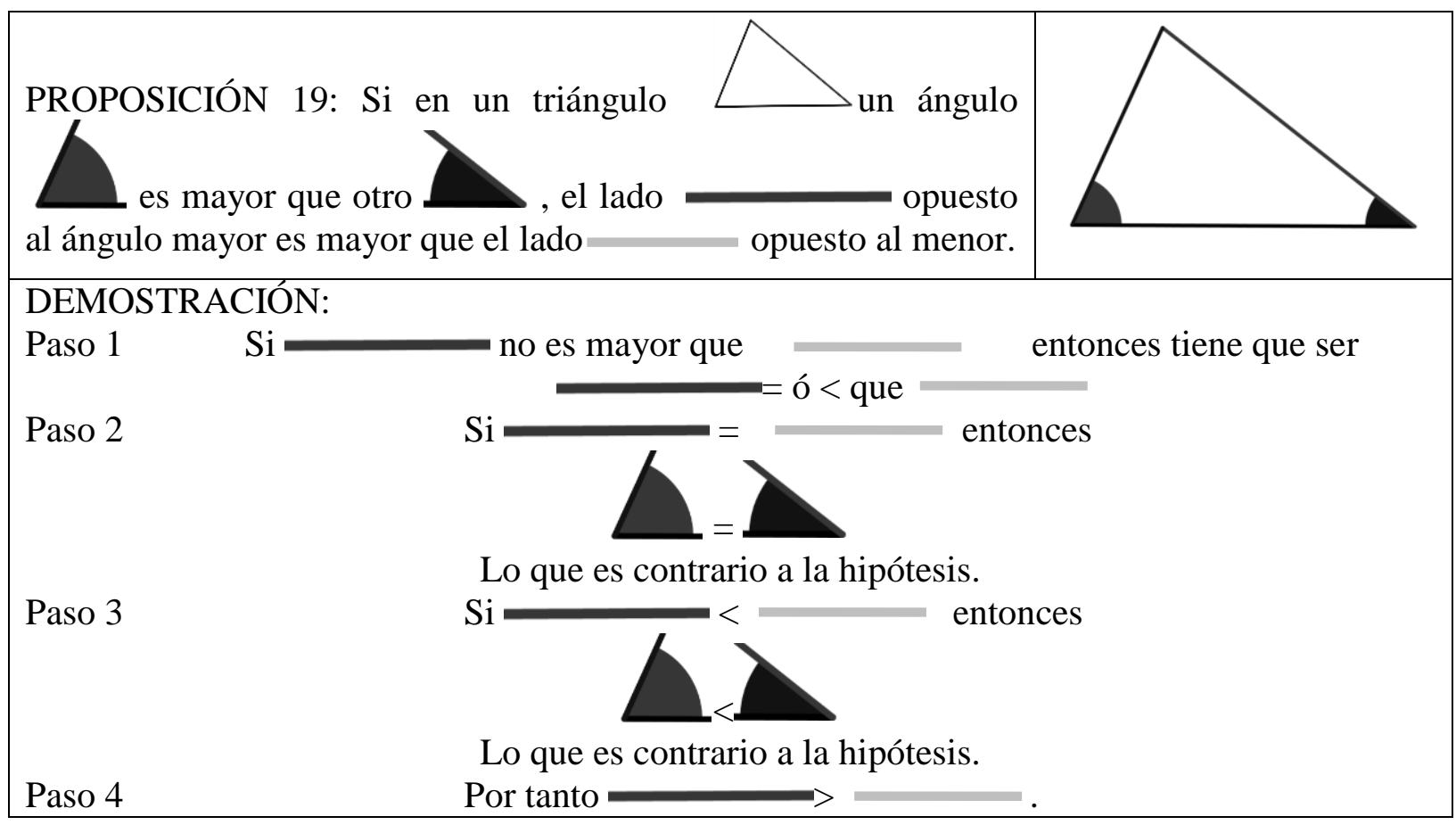

TAREA 2 ¿Aprecias alguna diferencia entre las formas de razonar que se emplean en las dos demostraciones anteriores? Coméntalas.

TAREA 3. Reescribe la Demostración de la proposición 20 mediante reducción al absurdo. PROPOSICIÓN 20:

Dos lados cualesquiera ( $\mathrm{y}$ ) de un triángulo

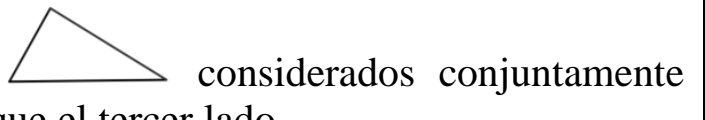
son mayores que el tercer lado

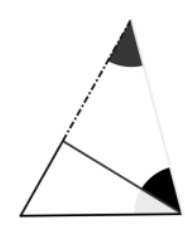

DEMOSTRACIÓN:

Prolonga y construye $= \pm n=n-n=$ dibuja

Como

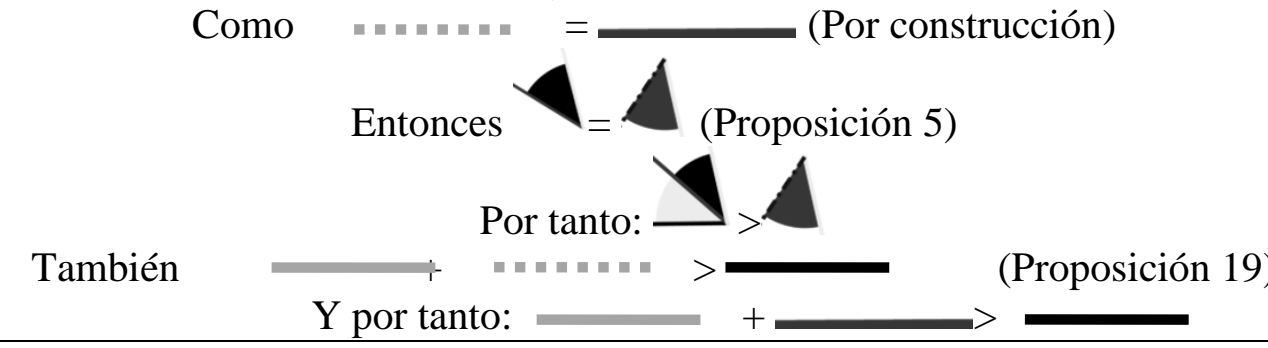
(Por construcción)

TAREA 4: Supón que debes explicar en clase la demostración de la proposición anterior. Construye una animación con GeoGebra y utilízala para apoyar tu explicación verbal. Escribe un guión de tu intervención en el que se muestre cómo combinas el uso del software con tus explicaciones.

TAREA 5. Centrándote en algún aspecto concreto del currículo de Bachillerato (Álgebra, Análisis...), diseña una actividad relacionada con la demostración e indica cómo la evaluarías 